\title{
Magnetically Tunable Rectangular Waveguide $E$-Plane Integrated Circuit Filters
}

\author{
JAROSLAW UHER, JENS BORNEMANN, AND FRITZ ARNDT, SENIOR MEMBER, IEEE
}

\begin{abstract}
A}$ rigorous field theory method is described for the computer-aided design of magnetically tunable $E$-plane metal insert filters, where the waveguide sections are symmetrically loaded with ferrite slabs, and for large-gap finline filters on a ferrite substrate. The design method is based on field expansion in suitably normalized eigenmodes which yields directly the modal scattering matrix of all discontinuities. The theory includes both higher order mode interaction and finite thickness of the metal inserts. Optimized data are given for magnetically tunable $K \boldsymbol{u}$-band metal insert and finline filter examples. The metal insert type achieves a tuning range of its operating midband from about 14.1 to $15.7 \mathrm{GHz}$. The theory is verified by measurements.
\end{abstract}

\section{INTRODUCTION}

$\mathrm{M}$ AGNETICALLY tunable waveguide bandpass filters, with the desired property that the filter response shape will remain more or less constant as the filter is tuned, are of considerable practical interest for many applications [1]-[4]. Useful techniques include ferrimagnetic YIG resonators [1], [2], or ferrite-slab-loaded evanescent-mode waveguide filters [3], [4]. This paper introduces magnetically tunable $E$-plane integrated circuit filters which are particularly appropriate for low-cost photolithographic fabrication techniques and for millimeterwave applications. These are the $E$-plane metal insert filter with lateral ferrite inserts (Fig. 1) and the large-gap finline filter on a ferrite substrate (Fig. 2). The operating band of the filters is controlled by the dc magnetic field on the ferrite. The design combines the well-known advantages of $E$-plane integrated circuit filters [5]-[14] with those of magnetically tunable ferrite-slab-loaded resonator sections [3], [4].

Many refined design methods for printed $E$-plane circuit filters without ferrite-loaded waveguide sections [5]-[14] and for ferrite-slab-loaded waveguides [15]-[21] are available. For the computer-aided design of the magnetically tunable $E$-plane integrated circuit filters presented in this paper (Figs. 1 and 2), the modal-S-matrix method [7], [9], [14], [22], [23] is applied, which has already proved to be highly appropriate for the accurate design of $E$-plane integrated components, since higher order mode coupling effects as well as the finite thickness of the inserts are included.

The structure under consideration (Figs. 1 and 2) is simply decomposed into appropriate key building blocks:

\footnotetext{
Manuscript received August 18, 1987: revised January 4, 1988.

J. Uher and F. Arndt are with the Microwave Department, University of Bremen, Kufstemer Str NW1, D-2800 Bremen 33, West Germany

J. Bornemann was with the Microwave Department, University of Bremen, Bremen, West Germany. He is now with the University of Victoria, Victoria, B.C., Canada.

IEEE Log Number 8821070 .
}

rectangular waveguide, ferrite-slab-loaded septate waveguide section (Fig. 1(b)), and ferrite-slab-loaded resonator section (Fig. 1(c)); rectangular waveguide, ferrite-slabloaded waveguide section with (Fig. 2(b)) and without two septa (Fig. 2(c)). The overall scattering matrix of the total filter component is calculated by suitable direct combination [7], [9], [14], [22], [23] of all single modal scattering matrices of the step discontinuities and homogeneous sections involved. This procedure preserves numerical accuracy, as the expressions contain exponential functions with only negative arguments. Moreover, no symmetry of the number of ports or modes at the discontinuities is required. This fact allows one to maintain the same algebraic procedures [7], [9], [14], [22], [23] for multiport discontinuities and offers the possibility of modifying adequately the number of modes [24], [25], if necessary, along the structure to be considered. The utilization of this nonsymmetry helps to circumvent the relative convergence problem [26] and to reduce computing time and storage requirements.

For computer optimization, the evolution strategy method [7], [9], [14], [27], i.e., a suitably modified directsearch procedure, is applied where no differentiation step in the optimization process is necessary; hence the problem of local minima may be avoided. Data for optimized two- and three-resonator magnetically tunable metal insert and finline filters for $\mathrm{Ku}$-band waveguide housings (15.799 $\mathrm{mm} \times 7.899 \mathrm{~mm}$ ) and commercially available TTI-2800, TTVG-1200, and RF-8 ferrite materials are given. The theory is experimentally verified through measured results.

\section{THEORY}

The modal $S$ matrix of the step discontinuity to the ferrite-slab-loaded waveguide (Fig. 2(c)) has already been derived in [21], and the procedure for obtaining the overall $S$ matrix of the filter structures is available in [7], [9], [14], and [21]-[23]. Therefore, for the field theory design of the filters presented in this paper (Figs. 1 and 2), only the derivation of three new modal $S$ matrices is required: $\left(S^{C}\right)$ of the coupling region of the $E$-plane metal insert filter with the lateral ferrite slabs (Fig. 1(b)), $\left(S^{R}\right)$ of the resonator region of the $E$-plane metal insert filter with the lateral ferrite slabs (Fig. 1(c)), and $\left(S^{F}\right)$ of the coupling region of the finline filter on a ferrite substrate (Fig. 2(b)).

For each homogeneous subregion, $v=\mathrm{I}$ to IV (Figs. 1(b), 1(c), 2(b)), the field equations [16] of the resulting $\mathrm{TE}_{n 0}$ wave, if a $\mathrm{TE}_{m 0}$ wave is incident, are derived from the electric field component $\vec{e}_{y} E_{y}^{(v)}$. This component is expressed as a sum of $N$ eigenmodes [15], [21], [29] satisfying 


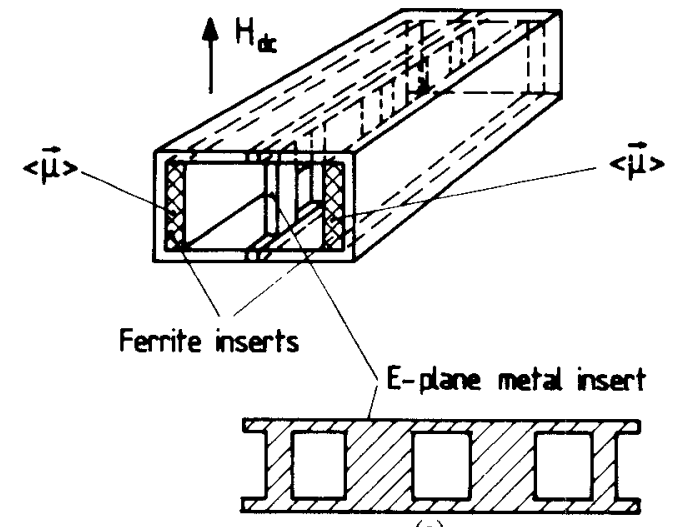

(a)
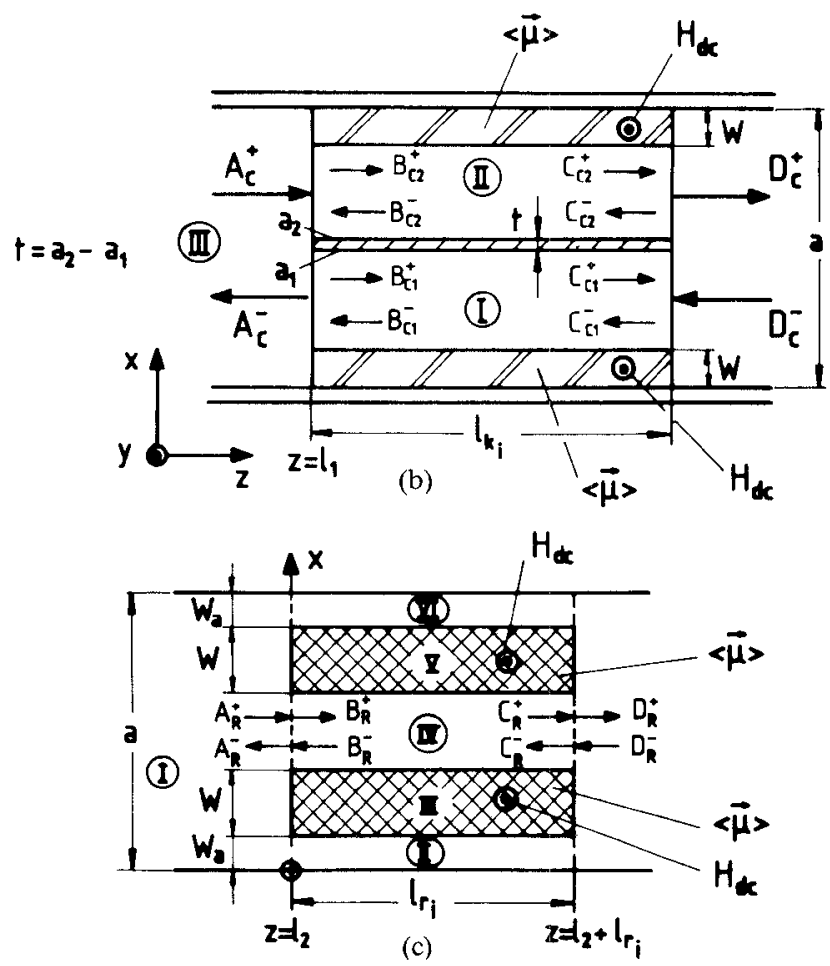

Fig. 1. Magnetically tunable $E$-plane metal insert filter with ferriteslab-loaded waveguide sections. (a) Complete filter structure. (b) Coupling region of finite length. (c) Resonator region of finite length; the air gap width $w_{a}$ due to fabrication tolerances is considered by the cross-section eigenvalue solution of the five-layer structure.

the vector Helmholtz equation and the boundary conditions at the discontinuities in the $x$ direction. For a dc magnetic field $H_{\mathrm{dc}}$ in the $y$ direction, the permeability tensor takes the form [15], [30], [31]

$$
\langle\vec{\mu}\rangle=\left(\begin{array}{ccc}
\mu_{1} & 0 & -j \kappa \\
0 & \mu_{r} & 0 \\
j \kappa & 0 & \mu_{1}
\end{array}\right)
$$

with elements $\mu_{1}, \mu_{r}$, and $\kappa$ given in [30] and [31]. For a demagnetized ferrite substrate (i.e., $H_{\mathrm{dc}}=0$ ), the off-diagonal tensor elements vanish, and the diagonal elements may be approximated by expressions given in [28]. The propagation factor $\gamma_{n}$ in the waveguide sections is determined via field matching [16], [21] of the transverse field components along the boundaries in the $x$ direction, together with the relations for the single wavenumbers in the

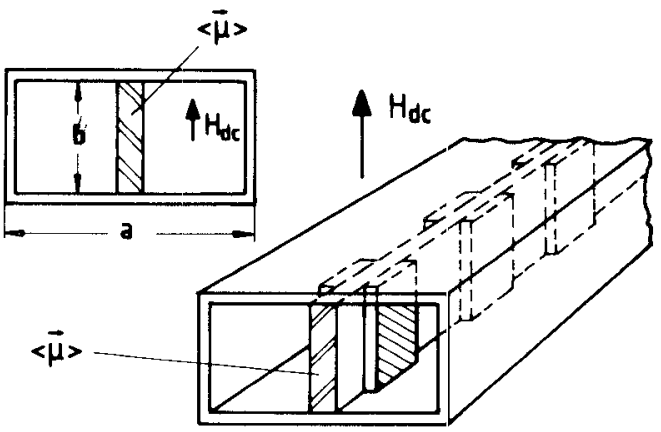

(a)

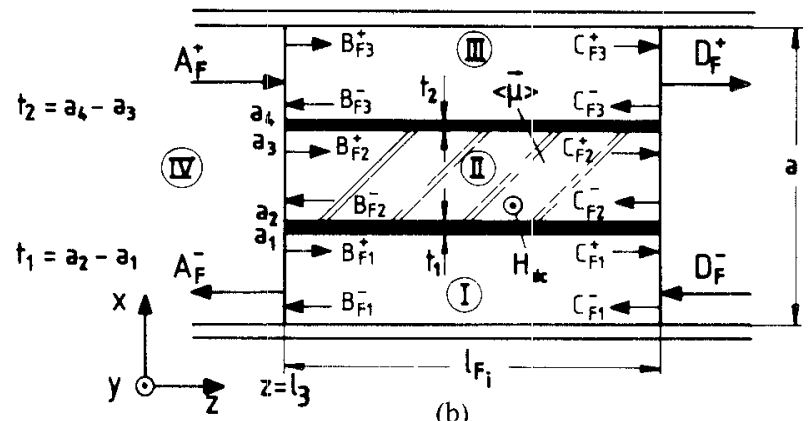

(b)

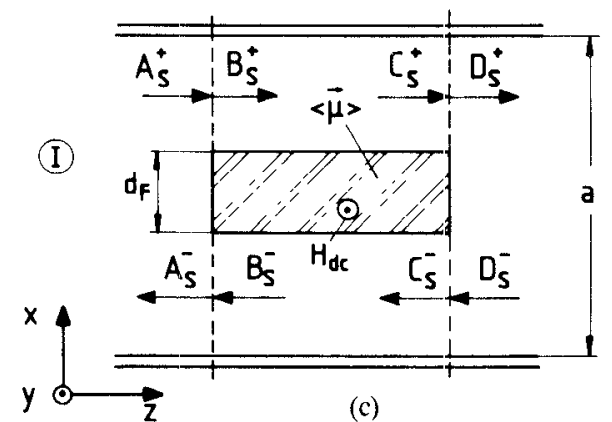

Fig. 2. Magnetically tunable large-gap finline filter on a ferrite substrate. (a) Complete filter structure. (b) Coupling region of finite length. (c) Resonator region of finite length.

cross-sectional subregions. The requirement that the system determinant be zero results in a transcendental equation for $\gamma_{n}$ which is solved numerically [21].

The influence of small lateral air gaps of width $w_{a}$ (cf. Fig. 1(c)), due to fabrication tolerances, on the filter response, which has been observed at evanescent-mode filters as well [4], is taken into account adequately by solving the scattering problem of a five-layer resonator region of length $l_{r l}$ (Fig. 1(c)), analogously to [21]. The effect of the air gaps on the evanescent modes in the coupling region (Fig. 1(b)), however, has turned out to be negligible. Therefore, for simplicity, the complicated $S$-matrix formulation of the corresponding septated double three-layer coupling structure (including $w_{a}$ ) is not presented here; the description is abbreviated by taking $w_{a}=0$ directly into account for the scattering matrix expression of the coupling structure.

The biorthogonality relations [16], [29] for anisotropic media are given by

$$
\left(\gamma_{n}+\gamma_{m}^{\prime}\right) \iint_{F}\left[\left(\vec{E}_{n} \times \vec{H}_{m}^{\prime}-\vec{E}_{m}^{\prime} \times \vec{H}_{n}\right) \cdot \vec{e}_{z}\right] d F=0 .
$$


The reciprocity principle involves the fields $E_{m}^{\prime}, H_{m}^{\prime}$, and the propagation constant $\gamma_{m}^{\prime}$ which are solutions of the related problem with the media characterized by the transposed permeability tensor [16].

A. Modal S Matrix $\left(S^{C}\right)$ of the Coupling Region of the Metal Insert Filter with Lateral Ferrite Slabs (cf. Fig. I(b))

Matching of the transversal field components at the corresponding interfaces (Fig. 1(b)) at $z=l_{1}$ yields

$$
\begin{aligned}
& \sum_{m=1}^{M}\left(A_{C m}^{+}+A_{C m}^{-}\right) T_{C m} u_{C m} \\
& \quad=\sum_{n=1}^{N}\left(B_{C 1 n}^{+} e_{1 n}^{+}+B_{C 1 n}^{-} e_{1 n}^{-}+B_{C 2 n}^{+} e_{2 n}^{+}+B_{C 2 n}^{-} e_{2 n}^{-}\right) \\
& \sum_{m=1}^{M}\left(A_{C m}^{+}-A_{C m}^{-}\right) T_{C m} Y_{C m} u_{C m} \\
& \quad= \begin{cases}\sum_{n=1}^{N}\left(B_{C 1 n}^{+} h_{1 n}^{+}-B_{C 1 n}^{-} h_{1 n}^{-}\right), & x \in(\mathrm{I}) \\
\sum_{n=1}^{N}\left(B_{C 2 n}^{+} h_{2 n}^{+}-B_{C 2 n}^{-} h_{2 n}^{-}\right), & x \in(\mathrm{II})\end{cases}
\end{aligned}
$$

where $T_{C m}$ is the normalization factor so that the power carried by a given wave is $1 \mathrm{~W}$ for a wave amplitude of $1 \sqrt{\mathrm{W}}$ [16]. The cross-section function $u_{C m}$, the wave admittance $Y_{C m}$, and the normalized eigenfunctions of the $E_{y}$ and $H_{x}$ component of the forward $(+)$ or reverse $(-) n$th mode, $e^{+}, e^{-}, h^{+}$, and $h^{-}$, respectively, are elucidated in the Appendix.

The general modal scattering matrix $\left(S^{C}\right)$ of the coupling structure of finite length $l_{k l}$ (Fig. 1(c)) is obtained by suitably combining the $S$ matrices involved:

$$
\left(\begin{array}{l}
\left(A_{C}^{-}\right) \\
\left(D_{C}^{+}\right)
\end{array}\right)=\left(\begin{array}{ll}
\left(S_{11}^{C}\right) & \left(S_{12}^{C}\right) \\
\left(S_{21}^{C}\right) & \left(S_{22}^{C}\right)
\end{array}\right) \cdot\left(\begin{array}{c}
\left(A_{C}^{+}\right) \\
\left(D_{C}^{-}\right)
\end{array}\right)
$$

where the coefficients are given in the Appendix.

\section{B. Modal $S$ Matrix $\left(S^{R}\right)$ of the Resonator Region of the Metal Insert Filter with Lateral Ferrite Slabs (cf. Fig. I(c))}

Matching of the transversal field components at the interfaces at $z=l_{2}$ (Fig. 1(c)) yields equations which are similar to (3). The corresponding cross-sectional eigenvalue problem leads to the transcendental equation given in the Appendix. The general scattering matrix $\left(S^{R}\right)$ of the resonator region of finite length $l_{r l}$ is given by

$$
\left(\begin{array}{c}
\left(A_{R}^{-}\right) \\
\left(D_{R}^{+}\right)
\end{array}\right)=\left(\begin{array}{ll}
\left(S_{11}^{R}\right) & \left(S_{12}^{R}\right) \\
\left(S_{21}^{R}\right) & \left(S_{22}^{R}\right)
\end{array}\right) \cdot\left(\begin{array}{c}
\left(A_{R}^{+}\right) \\
\left(D_{R}^{-}\right)
\end{array}\right)
$$

where the coefficients are also given in the Appendix.

C. Modal $S$ Matrix $\left(S^{F}\right)$ of the Coupling Region of the Finline Filter on a Ferrite Substrate (Fig. 2(b))

Field matching at $z=l_{3}$ (Fig. 2(b)) and combination of the related $S$ matrices involved leads to the general modal scattering matrix $\left(S^{F}\right)$ of the coupling region of finite

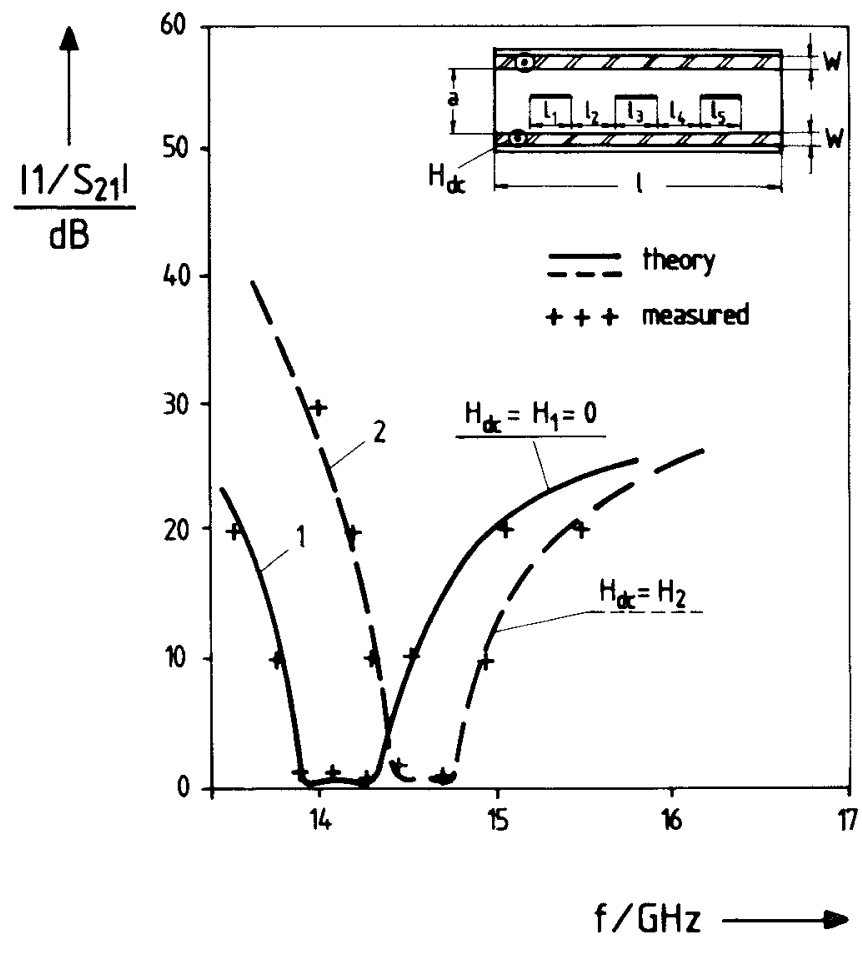

(a)

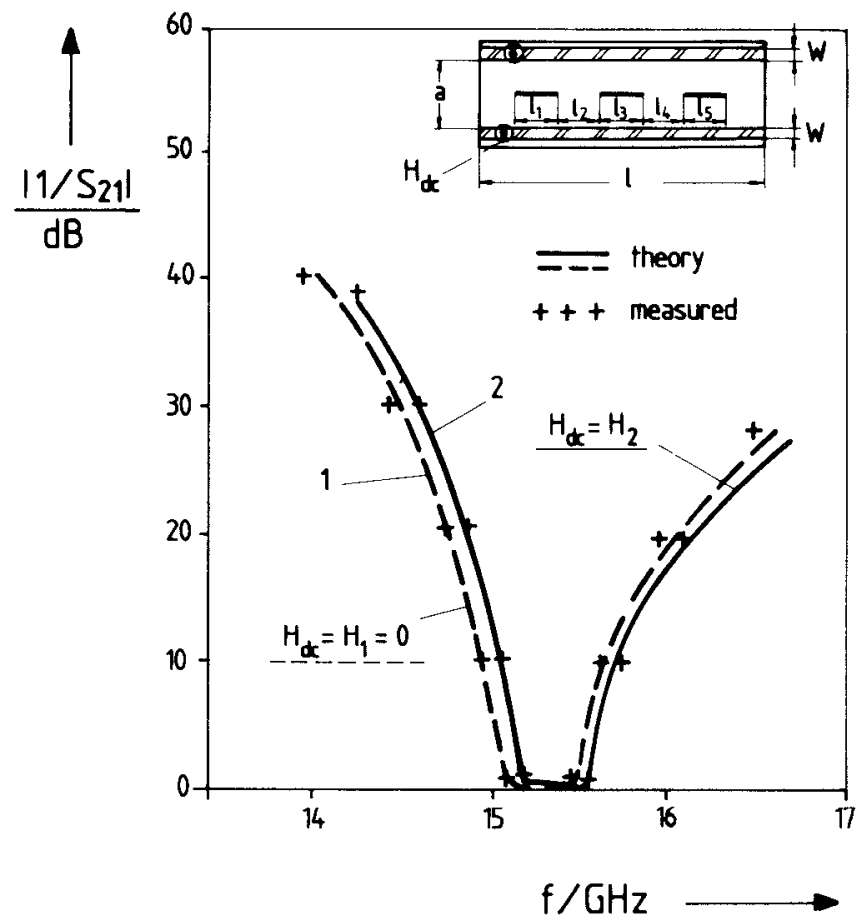

(b)

Fig. 3. Filter response of a magnetically tunable two-resonator $E$-plane metal insert filter with lateral ferrite TTI-2800 slabs of length $l=50$ $\mathrm{mm}$. Waveguide dimensions: $a=15.799 \mathrm{~mm}, b=\mathrm{a} / 2$, Filter data: $l_{1}=l_{5}=3.263 \mathrm{~mm}, l_{2}=l_{4}=8.914 \mathrm{~mm}, l_{3}=8.578 \mathrm{~mm}, t=0.19 \mathrm{~mm}$ - -..- theory, air gap width $w_{a}=0.1 \mathrm{~mm}$ (cf. Fig. $1(\mathrm{c})$ ) considered; ++++ measured. (a) Computer-optimized filter with ferrite slab width $w=1 \mathrm{~mm}$ de field strengths: $H_{1}=0$ (curve 1); $H_{2}=5.6 \cdot 10^{4}$ $\mathrm{A} / \mathrm{m}$ (curve 2). (b) Same filter but with reduced ferrite slab width $w=0.5 \mathrm{~mm}$ of same length. $H_{1}=0$ (curve 1 ); $H_{2}=1.72 \cdot 10^{5} \mathrm{~A} / \mathrm{m}$ (curve 2). 
length $l_{F i}$ :

$$
\left(\begin{array}{c}
\left(A_{F}^{-}\right) \\
\left(D_{F}^{+}\right)
\end{array}\right)=\left(\begin{array}{ll}
\left(S_{11}^{F}\right) & \left(S_{12}^{F}\right) \\
\left(S_{21}^{F}\right) & \left(S_{22}^{F}\right)
\end{array}\right) \cdot\left(\begin{array}{c}
\left(A_{F}^{+}\right) \\
\left(D_{F}^{-}\right)
\end{array}\right)
$$

where the coefficients are again given in the Appendix.

The overall scattering matrices of the complete filters are calculated by directly combining the appropriate single scattering matrices as shown in [7], the lengths of the intermediate homogeneous waveguide sections (III in Fig. 1(b), I in Figs. 1(c) and 2(c), IV in Fig. 2(b)) being reduced to zero for calculating the step discontinuities from one loaded waveguide section to another.

For computer optimization [7] of the filters, the expansion into ten eigenmodes at each discontinuity has turned out to be sufficient. The final design data are proven through an expansion of 30 eigenmodes. The convergence behavior of the modal method used for ferrite-loaded waveguides has already been demonstrated in [21].

\section{RESULTS}

Fig. 3(a) shows the calculated and measured filter response of a computer-optimized two-resonator magnetically tunable metal insert filter with lateral ferrite TTI-2800 slabs (Transtech Inc.) of widths $w=1 \mathrm{~mm}$ for two different dc field strengths. The operating midband of the filter may be tuned from about $14.2 \mathrm{GHz}$ to $14.6 \mathrm{GHz}$. The influence of the ferrite slab width $w$ on the tuning bandwidth is demonstrated by Fig. 3(b), where the filter response of the filter with the same dimensions but with lateral ferrite slabs of reduced width $w=0.5 \mathrm{~mm}$ is plotted against frequency. An air gap width $w_{a}=0.1 \mathrm{~mm}$ (cf. Fig. 1(c)) between the ferrite slabs and the waveguide narrow walls is included in the calculations. Good agreement between theory and measured results may be stated.

A computer-optimized three-resonator magnetically tunable $E$-plane metal insert filter with lateral ferrite TTI2800 slabs of width $w=1 \mathrm{~mm}$ (Fig. 4(a)) achieves a tuning range of the operating midband frequency from about 14.1 to $15.7 \mathrm{GHz}$. To demonstrate the significance of the influence of an air gap of width $w_{a}$ between the lateral ferrite slabs and the narrow walls of the waveguide on the filter response of a magnetically tunable metal insert filter, Fig. 4(b) shows the calculated insertion loss in decibels of a three-resonator filter for two different air gap widths together with the measured results. If the actual air gap width $w_{a}=0.1 \mathrm{~mm}$ due to fabrication tolerances is taken into account (curve 1), good agreement between theory and measurements may be observed.

Figs. 5 and 6 show the filter response of computer-optimized magnetically tunable three-resonator large-gap finline filters on ferrite substrates RF-8 (AEG/Telefunken) and TTVG-1200 (Transtech. Inc.), respectively. These materials have turned out to be more appropriate for this filter type than the TTI-2800 substrate, which has been chosen for the metal insert filters. Compared with ferrite-

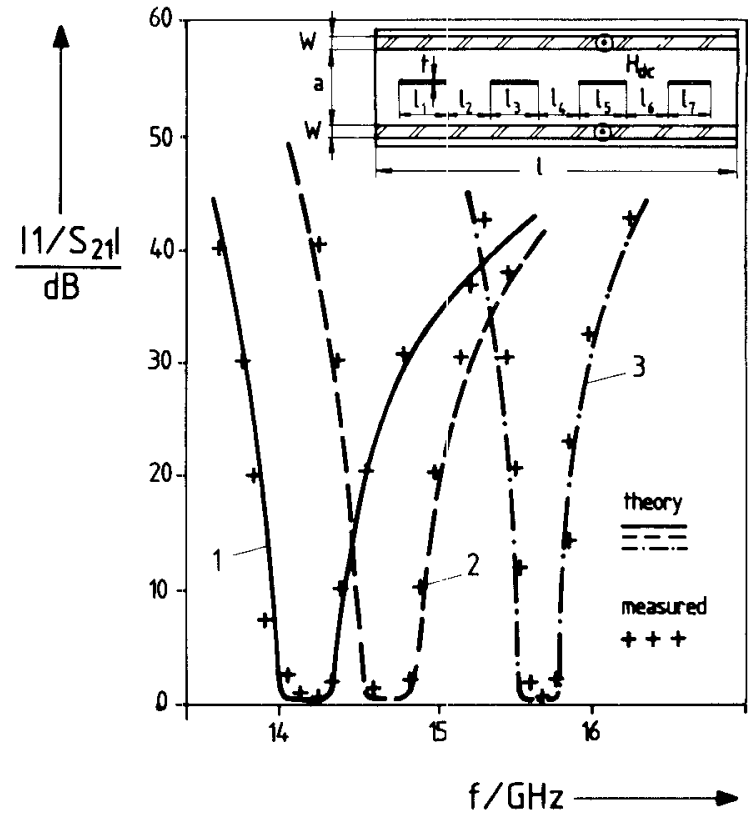

(a)

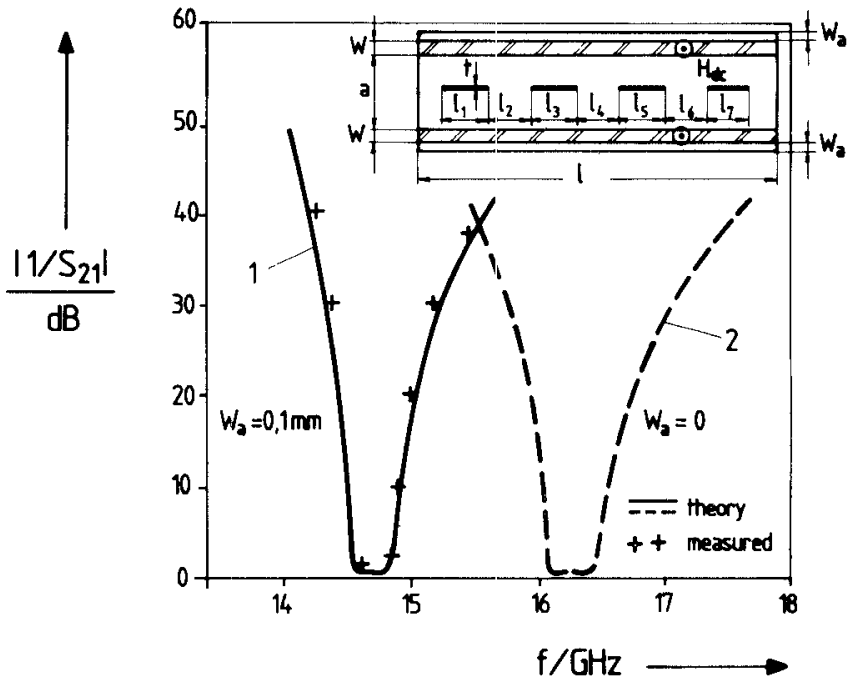

(b)

Fig. 4. Filter response of a magnetically tunable three-resonator metal insert filter with lateral ferrite TTI-2800 slabs of width $w=1 \mathrm{~mm}$, length $l=50 \mathrm{~mm}$. Waveguide dimensions: $a=15.799 \mathrm{~mm}, b=\mathrm{a} / 2$. Filter data: $l_{1}=l_{7}=3.590 \mathrm{~mm}, l_{2}=l_{6}=8.916 \mathrm{~mm}, l_{3}=l_{5}=9.417$ $\mathrm{mm}, l_{4}=8.921 \mathrm{~mm}, t=0.19 \mathrm{~mm}$. (a) Three different dc field strengths: $H_{1}=0$ (curve 1 ), $H_{2}=4.8 \cdot 10^{4} \mathrm{~A} / \mathrm{m}$ (curve 2 ), $H_{3}=1.72 \cdot 10^{5} \mathrm{~A} / \mathrm{m}$ (curve 3 ); — , ............ theory, air gap width $w_{a}=0.1 \mathrm{~mm}$ (cf. Fig. 1(c)) considered. (b) Influence of the air gap width $w_{a}$. Curve 1: $w_{a}=0.1 \mathrm{~mm}$ considered; curve $2: w_{a}$ neglected $\left(w_{a}=0\right) . H_{\mathrm{dc}}=4.8 \cdot 10^{4}$ $\mathrm{A} / \mathrm{m}$.

loaded metal insert filters (cf. e.g. Fig. 4(a)), however, the tuning range of the operating midband frequencies (Figs. 5 and 6) is smaller for the finline type. This is caused by two facts: the effective ferrite slab thickness within the resonator regions may be made larger for metal insert filters, and the relative wavelength variation due to an appropriate dc magnetic field, for off-center slab positions, is higher than for central locations. 


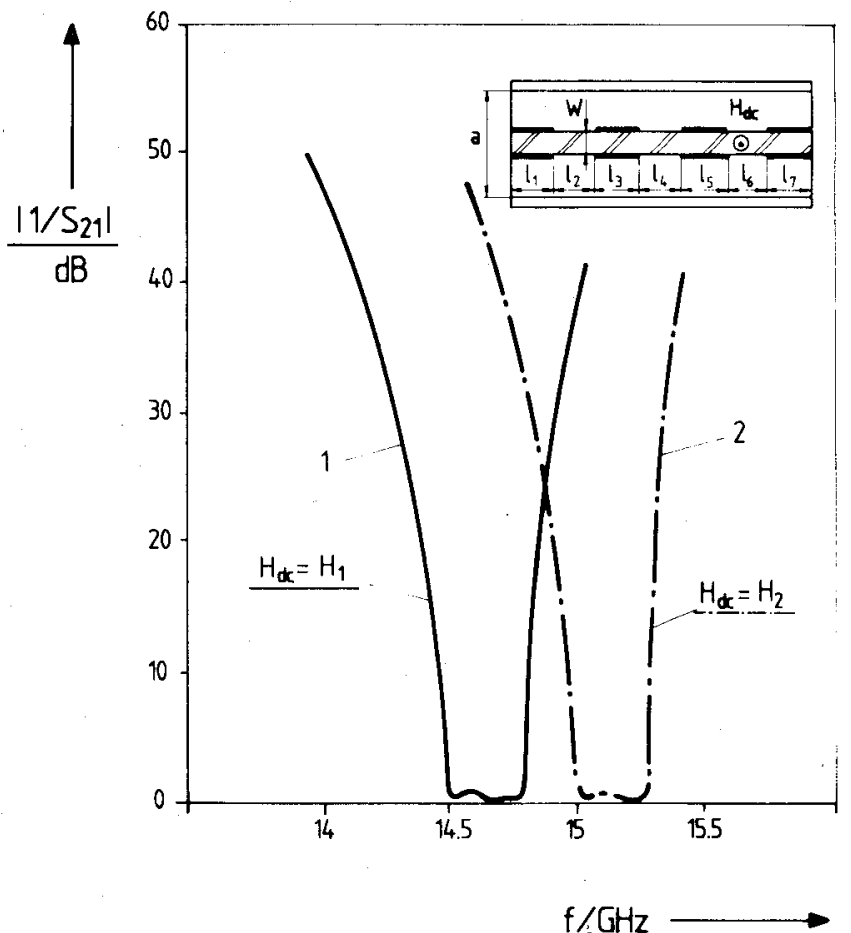

Fig. 5. Filter response of a magnetically tunable three-resonator largegap finline filter on a ferrite RF-8 substrate of width $w=1 \mathrm{~mm}$. Waveguide dimensions: $a=15.799 \mathrm{~mm}, b=a / 2$. Filter data: $l_{1}=l_{7}=$ $3.460 \mathrm{~mm}, l_{2}=l_{6}=4.836 \mathrm{~mm}, l_{3}=l_{5}=7.927 \mathrm{~mm}, l_{4}=4.873 \mathrm{~mm}$. dc field strengths: $H_{1}=0$ (curve 1 ), $H_{2}=1.4 \cdot 10^{5} \mathrm{~A} / \mathrm{m}$ (curve 2).

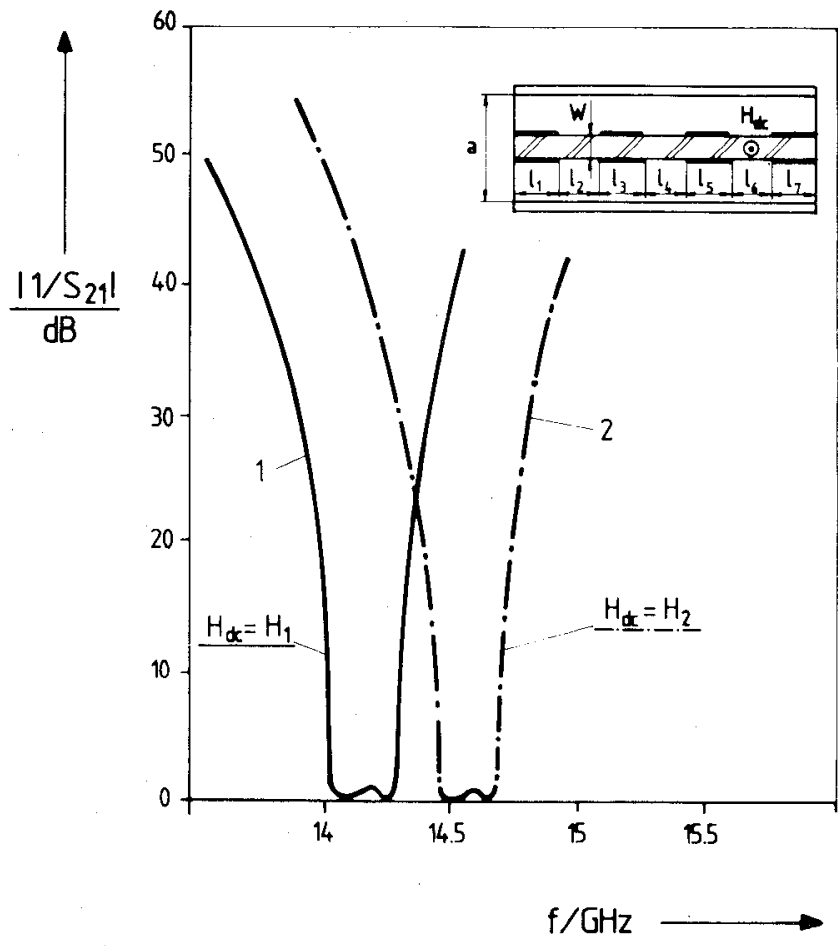

Fig. 6. Filter response of a magnetically tunable three-resonator largegap finline filter on a ferrite TTVG-1200 substrate of width $w=0.7$ $\mathrm{mm}$. Waveguide dimensions: $a=15.799 \mathrm{~mm}, b=a / 2$. Filter data: $l_{1}=l_{7}=3.457 \mathrm{~mm}, l_{2}=l_{6}=4.836 \mathrm{~mm}, l_{3}=l_{5}=8.572 \mathrm{~mm}, l_{4}=4.817$ mm. dc field strengths: $H_{1}=0$ (curve 1 ), $H_{2}=2.2 \cdot 10^{5} \mathrm{~A} / \mathrm{m}$ (curve 2).

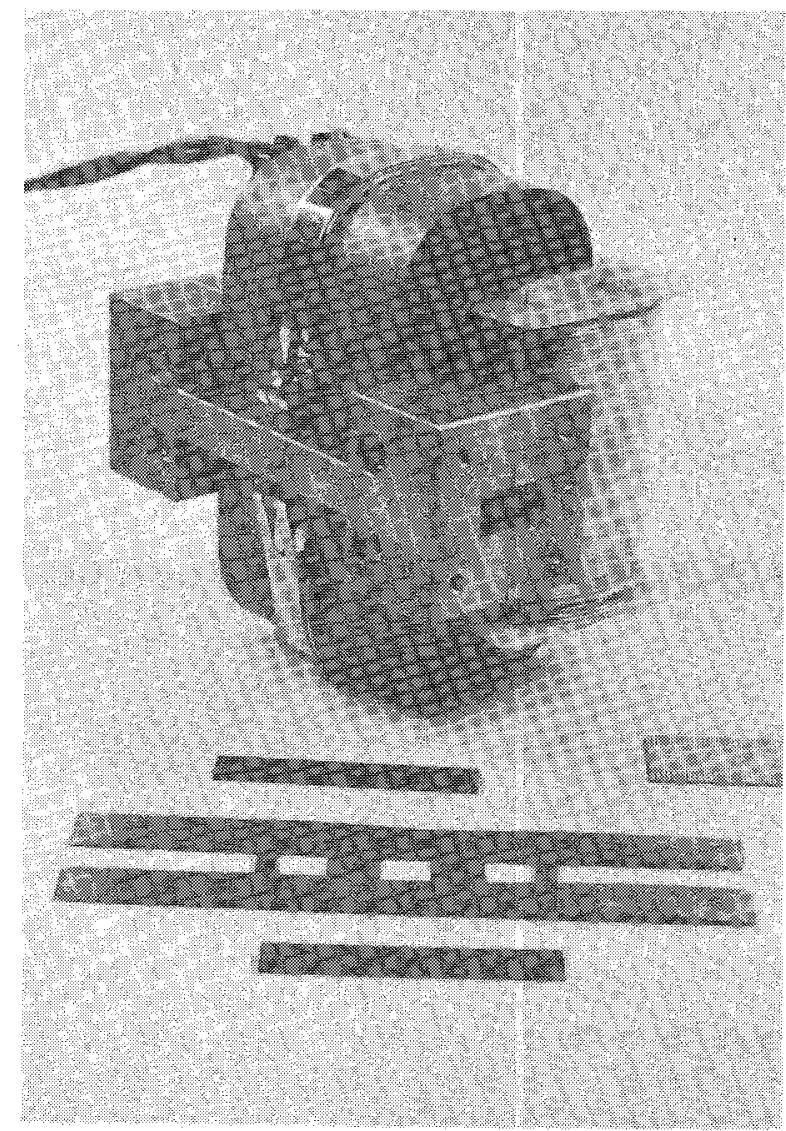

Fig. 7. Components of a fabricated magnetically tunable $E$-plane metal insert filter: R140 waveguide housing together with the biasing magnet, the photoetched $0.19 \mathrm{~mm}$ thick metal insert, and the two lateral ferrite slab inserts.

The components of the magnetically tunable three-resonator $E$-plane metal insert filter which were fabricated to provide the measured results presented in Fig. 4 are shown in Fig. 7: the R140 waveguide housing $(15.799 \mathrm{~mm} \times 7.899$ $\mathrm{mm})$ together with the biasing magnet, the photoetched $0.19 \mathrm{~mm}$ thick metal insert (99.9 percent pure copper), and the two lateral TTI-2800 ferrite slabs of standard width $w=1 \mathrm{~mm}$. The biasing dc field was measured with a gaussmeter; the internal magnetic field was calculated according to the procedure given in [32]. For the measurements, an HP 8410 network analyzer was used [21].

\section{Conclusions}

A rigorous field theory is presented for the optimum design of ferrite-loaded magnetically tunable $E$-plane metal insert filters and large-gap finline filters on a ferrite substrate. The design combines the advantages of $E$-plane integrated circuit filters with the tuning capability of ferrite-loaded resonator sections. As all relevant parameters, such as higher order mode interactions and the influence of an additional air gap between ferrite slabs and the waveguide walls, are included in the design theory, the theoretically predicted values agree well with measured results. 


\section{APPENDIX}

\section{A. Abbreviations in (3)}

$T_{c m}=$ normalization factor so that the power carried by a given wave is $1 \mathrm{~W}$ for a wave amplitude of $\sqrt{1 \mathrm{~W}}$.

$$
\begin{aligned}
& u_{c m}=\sin \left(\frac{m \pi}{a} x\right) \\
& e_{1 n}^{+}= \begin{cases}E_{1 n}^{(\mathrm{I})+} \sin \left[k_{F n}^{(\mathrm{I})+} x\right], & x \in(0, w) \\
E_{2 n}^{(\mathrm{I})+} \sin \left[k_{L n}^{(\mathrm{I})+}\left(a_{1}-x\right)\right], & x \in\left(w, a_{1}\right)\end{cases} \\
& e_{2 n}^{+}= \begin{cases}E_{1 n}^{(\mathrm{II})+} \sin \left[k_{L n}^{(\mathrm{II})+}\left(x-a_{2}\right)\right], & x \in\left(a_{2}, a-w\right) \\
E_{2 n}^{(\mathrm{II})+} \sin \left[k_{F n}^{(\mathrm{II})+}(a-x)\right], & x \in(a-w, a)\end{cases}
\end{aligned}
$$

$$
h_{1 n}^{+}= \begin{cases}\frac{j \gamma_{n}^{(\mathrm{I})+} E_{1 n}^{(\mathrm{I})+}}{\omega \mu_{0} \mu_{\mathrm{eff}}} \sin \left[k_{F n}^{(\mathrm{I})+} x\right] & \\ -\frac{E_{1 n}^{(\mathrm{I})+}\left(\frac{\kappa}{\mu_{1}}\right) k_{F n}^{(\mathrm{I})+}}{\omega \mu_{0} \mu_{\mathrm{eff}}} \cos \left[k_{F n}^{(\mathrm{I})+} x\right], & \\ \frac{j \gamma_{n}^{(\mathrm{I})+} E_{2 n}^{(\mathrm{I})+} \sin \left[k_{L n}^{(\mathrm{I})+}\left(a_{1}-x\right)\right],}{\omega \mu_{0}} & x \in(w, w) \\ & x \in\left(w, a_{1}\right)\end{cases}
$$

$$
h_{2 n}^{+}=\left\{\begin{array}{c}
\frac{j \gamma_{n}^{(\mathrm{II})+} E_{1 n}^{(\mathrm{II})+}}{\omega \mu_{0}} \sin \left[k_{L n}^{(\mathrm{II})+}\left(x-a_{2}\right)\right], \\
\quad x \in\left(a_{2}, a-w\right) \\
\frac{j \gamma_{n}^{(\mathrm{II})+} E_{2 n}^{(\mathrm{II})+} \sin \left[k_{F n}^{(\mathrm{II})+}(a-x)\right]}{\omega \mu_{0} \mu_{\mathrm{eff}}} \\
+\frac{E_{2 n}^{(\mathrm{II})+}\left(\frac{\kappa}{\mu_{1}}\right) k_{F n}^{(\mathrm{II})+}}{\omega \mu_{0} \mu_{\mathrm{eff}}} \cos \left[k_{F n}^{(\mathrm{II})+}(a-x)\right], \\
x \in(a-w, a)
\end{array}\right.
$$

$$
Y_{c m}=\frac{k_{z m}}{\omega \mu_{0}} \quad\left(k_{z m}\right)^{2}=k_{0}^{2}-\left(\frac{m \pi}{a}\right)^{2} \quad k_{0}^{2}=\omega^{2} \mu_{0} \epsilon_{0}
$$

$$
\begin{aligned}
\left(k_{L n}^{+}\right)^{2} & =\left(\gamma_{n}^{+}\right)^{2}+k_{0}^{2} \quad\left(k_{F n}^{+}\right)^{2}=\left(\gamma_{n}^{+}\right)^{2}+k_{0}^{2} \epsilon_{f} \mu_{\mathrm{eff}} \\
\mu_{\mathrm{eff}} & =\frac{\mu_{1}^{2}-\kappa^{2}}{\mu_{1}}
\end{aligned}
$$

$e_{1 n}^{-}, e_{2 n}^{-}, h_{1 n}^{-}, h_{2 n}^{-}$are given analogously to (A2)-(A5) by inclusion of the related wavenumbers $k^{-}$in the reverse $(-)$ direction in the corresponding subregions.

\section{B. Scattering Coefficients in (4)}

$$
\begin{aligned}
\left(S_{11}^{C}\right)=\left(S_{22}^{C}\right)= & \left\{(U)+(E)-(T)[(E)+(U)]^{-1}(T)\right\}^{-1} \\
& \cdot\left\{(T)-(U)-(E)[(E)+(U)]^{-1}(E)\right\} \\
\left(S_{12}^{C}\right)=\left(S_{21}^{C}\right)= & \left\{(U)+(E)-(T)[(E)+(U)]^{-1}(T)\right\}^{-1} \\
& \cdot(E)\left\{(U)-[(E)+(U)]^{-1}[(T)-(E)]\right\}
\end{aligned}
$$

with the matrix coefficients

$$
\begin{aligned}
(E)= & \left(L_{E}^{(\mathrm{I})+}\right)\left(W^{(\mathrm{I})}\right)\left[\left(L_{H}^{(\mathrm{I})-}\right)\left(R_{1}^{-}\right)\right]^{-1} \\
& +\left(L_{E}^{(\mathrm{I})-}\right)\left(R_{1}^{-}\right)\left(P^{(\mathrm{I})}\right)\left(L_{H}^{(\mathrm{I})+}\right)^{-1} \\
& -\left(L_{E}^{(\mathrm{I})-}\right)\left(P^{(\mathrm{I})}\right)\left[\left(L_{H}^{(\mathrm{I})+}\right)\left(R_{1}^{+}\right)\right]^{-1} \\
& -\left(L_{E}^{(\mathrm{I})+}\right)\left(R_{1}^{+}\right)\left(W^{(\mathrm{I})}\right)\left(L_{h}^{(\mathrm{I})-}\right)^{-1} \\
(T)= & \left(L_{E}^{(\mathrm{I})-}\right)\left(P^{(\mathrm{I})}\right)\left(L_{H}^{(\mathrm{I})+}\right)^{-1} \\
& +\left(L_{E}^{(\mathrm{I})+}\right)\left(R_{1}^{+}\right)\left(W^{(\mathrm{I})}\right)\left[\left(L_{H}^{(\mathrm{I})-}\right)\left(R_{1}^{-}\right)\right]^{-1} \\
& -\left(L_{E}^{(\mathrm{I})+}\right)\left(W^{(\mathrm{I})}\right)\left(L_{H}^{(\mathrm{I})-}\right)^{-1} \\
& -\left(L_{E}^{(\mathrm{I})-}\right)\left(R_{1}^{-}\right)\left(P^{(\mathrm{I})}\right)\left[\left(L_{H}^{(\mathrm{I})+}\right)\left(R_{1}^{+}\right)\right]^{-1}
\end{aligned}
$$

$(U)=$ unit matrix

$$
\begin{aligned}
\left(P^{(\mathrm{I})}\right)=\{ & {\left[\left(L_{H}^{(\mathrm{I})+}\right)\left(R_{1}^{+}\right)\right]^{-1}\left(L_{H}^{(\mathrm{I})-}\right) } \\
& \left.-\left(L_{H}^{(\mathrm{I})+}\right)^{-1}\left(L_{H}^{(\mathrm{I})-}\right)\left(R_{1}^{-}\right)\right\}^{-1} \\
\left(W^{(\mathrm{I})}\right)=\{ & {\left[\left(L_{H}^{(\mathrm{I})-}\right)\left(R_{1}^{-}\right)\right]^{-1}\left(L_{H}^{(\mathrm{I})+}\right) } \\
& \left.-\left(L_{H}^{(\mathrm{I})-}\right)^{-1}\left(L_{H}^{(\mathrm{I})+}\right)\left(R_{1}^{+}\right)\right\}^{-1}
\end{aligned}
$$

and

$$
\begin{gathered}
\left(L_{E}^{(\mathrm{I})+}\right)_{m k}=\left(D_{E m m}\right)^{-1}\left[E_{1 k}^{(\mathrm{I})+} I_{m k}^{(\mathrm{I})+}+E_{2 k}^{(\mathrm{I})+} I_{m k}^{(\mathrm{II})+}\right] \\
\left(L_{H}^{(\mathrm{I})+}\right)_{m k}=\left(D_{H m m}\right)^{-1}\left[\frac{j \gamma_{k}^{(\mathrm{I})+}}{\omega \mu_{0}} E_{2 k}^{(\mathrm{I})+} I_{m k}^{(\mathrm{II})+}+H_{k}^{(\mathrm{I})+} I_{m k}^{(\mathrm{I})+}\right. \\
\left.+G_{k}^{(\mathrm{I})+} J_{m k}^{(\mathrm{II})+}\right] \\
(\mathrm{A} 12) \\
G_{k}^{(\mathrm{I})+}=-\frac{E_{1 k}^{(\mathrm{I})+}\left(\frac{\kappa}{\mu_{1}}\right) k_{F k}^{(\mathrm{I})+}}{\omega \mu_{0} \mu_{\mathrm{eff}}} \quad H_{k}^{(\mathrm{I})+}=\frac{E_{1 k}^{(\mathrm{I})+} j \gamma_{k}^{(\mathrm{I})+}}{\omega \mu_{0} \mu_{\mathrm{eff}}} \\
(\mathrm{A} 13) \\
D_{E m m}=\frac{a}{2} T_{m} \quad D_{H m m}=\frac{a}{2} T_{m} Y_{m} \\
\left(R_{1}^{+}\right)_{k k}=\exp \left[-\gamma_{k}^{(\mathrm{I})+} \cdot l^{(C)}\right] .
\end{gathered}
$$


The coupling integrals are given by

$$
\begin{aligned}
& I_{m k}^{(\mathrm{I})+}=\int_{0}^{w} \sin \left[k_{F k}^{(\mathrm{I})+} x\right] u_{m}(x) d x \\
& I_{m k}^{(\mathrm{II})+}=\int_{w}^{a_{1}} \sin \left[k_{L k}^{(\mathrm{I})+}\left(a_{1}-x\right)\right] u_{m}(x) d x \\
& J_{m k}^{(\mathrm{I})+}=\int_{0}^{w} \cos \left[k_{F k}^{(\mathrm{I})+} x\right] u_{m}(x) d x .
\end{aligned}
$$

C. Transcendental Equation of the Cross-Section Eigenvalue Problem of the Five-Layer Structure (Fig. 1(c))

$$
\begin{aligned}
f\left(\gamma_{n}\right)= & \sin \left[k_{L n}\left(a-2 w-4 w_{a}\right)\right] \sin ^{2}\left(k_{F n} w\right) \\
& \cdot\left[\left(\frac{A_{L F}}{2}\right)^{2}-\frac{1}{2}\left\{\frac{\left.\left.\mu_{\mathrm{cff}}\left(\frac{\kappa}{\mu_{1}}\right) \gamma_{n}\right)^{2}\right]}{k_{L n}}\right\}\right] \\
& +\left[\frac{k_{F n} \mu_{\mathrm{eff}} \cos \left(k_{F n} w\right) \sin \left(k_{L n}\left(\frac{a}{2}-w\right)\right)}{k_{L n}}\right]^{2} \\
& -\left[\frac{A_{F L} \sin \left(k_{F n} w\right)}{2}\right]^{2} \sin \left[k_{L n}(a-2 w)\right] \\
& -\left[\frac{\sin \left(k_{F n} w\right)}{2}\right]^{2} A_{L F} A_{F L} \sin \left(2 k_{L n} w_{a}\right) \\
& +\frac{\mu_{\mathrm{eff}} k_{F n} A_{L F}}{4 k_{L n}} \sin \left(2 k_{F n} w\right) \sin \left[k_{L n}\left(\frac{a}{2}-w\right)\right] \\
& \cdot\left\{\sin \left[k_{L n}\left(\frac{a}{2}-w-2 w_{a}\right)\right]\right. \\
& \left.+\cos \left[k_{L n}\left(\frac{a}{2}-w-2 w_{a}\right)\right]\right\} \\
& \left.+\frac{\mu_{\mathrm{eff}} k_{F n} A_{F L}}{4 k_{L n}} \sin \left(2 k_{F n} w\right) \sin \left[k_{L n}\left(\frac{a}{2}-w\right)\right]-\sin \left[k_{L n}\left(\frac{a}{2}-w\right)\right]\right\} \\
& \left.\left.+k^{2}\right)\right]
\end{aligned}
$$

$$
\begin{aligned}
& A_{L F}=\mu_{\mathrm{eff}}^{2}+\frac{\gamma_{n}^{2}}{k_{L n}^{2}}\left(\frac{\kappa}{\mu_{1}}\right)^{2}-\left[\frac{k_{F n}}{k_{L n}}\right]^{2} \\
& A_{F L}=\mu_{\mathrm{eff}}^{2}-\frac{\gamma_{n}^{2}}{k_{L n}^{2}}\left(\frac{\kappa}{\mu_{1}}\right)^{2}+\left[\frac{k_{F n}}{k_{L n}}\right]^{2} .
\end{aligned}
$$

\section{Scattering Coefficients in (5)}

$$
\begin{aligned}
&\left(S_{11}^{R}\right)=\left(S_{22}^{R}\right)=\left(S_{11}^{0}\right)+\left(S_{12}^{0}\right)(R) \\
& \cdot\left[(U)-\left(S_{22}^{0}\right)(R)\left(S_{22}^{0}\right)(R)\right]^{-1} \\
& \cdot\left(S_{22}^{0}\right)(R)\left(S_{21}^{0}\right) \\
&\left(S_{12}^{R}\right)=\left(S_{21}^{R}\right)=\left(S_{12}^{0}\right)(R)\left[(U)-\left(S_{22}^{0}\right)(R)\left(S_{22}^{0}\right)(R)\right]^{-1} \cdot\left(S_{21}^{0}\right) \\
&\left(S_{11}^{0}\right)=\left(D_{E}\right)^{-1}\left(L_{E}\right) 2(M)-(U) \\
&\left(S_{12}^{0}\right)=\left(D_{E}\right)^{-1}\left(L_{E}\right)\{(U)+(M) \\
&\left.\cdot\left[\left(D_{H}\right)^{-1}\left(L_{H}\right)-\left(D_{E}\right)^{-1}\left(L_{E}\right)\right]\right\} \\
&\left(S_{21}^{0}\right)=2(M) \\
&\left(S_{22}^{0}\right)=2(M)\left(D_{H}\right)^{-1}\left(L_{H}\right)-(U) \\
&(M)=\left[\left(D_{E}\right)^{-1}\left(L_{E}\right)+\left(D_{H}\right)^{-1}\left(L_{H}\right)\right]^{-1} . \quad(\mathrm{A} 20)
\end{aligned}
$$

The elements of matrices $(R),\left(L_{E}\right)$, and $\left(L_{H}\right)$ are given by

$$
\begin{aligned}
(R)_{k k}= & \exp \left(-\gamma_{k} \cdot l_{R}\right) \\
\left(L_{E}\right)_{m k}= & E_{k}^{(\mathrm{II})} I_{m k}^{(\mathrm{I})}+E_{k}^{(\mathrm{III})} I_{m k}^{(\mathrm{II})}+F_{k}^{(\mathrm{III})} I_{m k}^{(\mathrm{III})}+E_{k}^{(\mathrm{IV})} I_{m k}^{(\mathrm{IV})} \\
\left(L_{H}\right)_{m k}= & E_{k}^{(\mathrm{II})} \frac{j \gamma_{k}}{\omega \mu_{0} \mu_{\mathrm{eff}}} I_{m k}^{(\mathrm{I})}-E_{k}^{(\mathrm{II})} \frac{\frac{\kappa}{\mu_{1}} k_{F k}}{\omega \mu_{0} \mu_{\mathrm{eff}}} J_{m k}^{(\mathrm{I})} \\
& +\frac{j \gamma_{k}}{\omega \mu_{0}}\left[E_{k}^{(\mathrm{III})} I_{m k}^{(\mathrm{II})}+F_{k}^{(\mathrm{III})} I_{m k}^{(\mathrm{III})}\right] \\
& +E_{k}^{(\mathrm{IV})} \frac{j \gamma_{k}}{\omega \mu_{0} \mu_{\mathrm{ef}}} I_{m k}^{(\mathrm{IV})} \\
& +E_{k}^{(\mathrm{IV})} \frac{\frac{\kappa}{\mu_{1}} k_{F k}}{\omega \mu_{0} \mu_{\mathrm{eff}}} J_{m k}^{(\mathrm{IV})}
\end{aligned}
$$

with the coupling integrals

$$
\begin{aligned}
I_{m k}^{(\mathrm{I})} & =\int_{0}^{w} \sin \left(k_{F k} x\right) u_{m}(x) d x \\
I_{m k}^{(\mathrm{II})} & =\int_{w}^{a-w} \sin \left(k_{L k} x\right) u_{m}(x) d x \\
I_{m k}^{(\mathrm{III})} & =\int_{w}^{a-w} \cos \left(k_{L k} x\right) u_{m}(x) d x \\
I_{m k}^{(\mathrm{IV})} & =\int_{a-w}^{a} \sin \left(k_{F k}(a-x)\right) u_{m}(x) d x \\
J_{m k}^{(\mathrm{I})} & =\int_{0}^{w} \cos \left(k_{F k} x\right) u_{m}(x) d x \\
J_{m k}^{(\mathrm{IV})} & =\int_{a-w}^{a} \cos \left(k_{F k}(a-x)\right) u_{m}(x) d x .
\end{aligned}
$$




\section{E. Scattering Coefficients in (6)}

$$
\begin{aligned}
\left(S_{11}^{F}\right)=\left(S_{22}^{F}\right)=- & \left\{(U)-[(U)-(E)]^{-1}(W)\right. \\
& \left.\cdot[(U)-(E)]^{-1}(W)\right\}^{-1} \\
& \cdot\left\{[(U)-(E)]^{-1}(W)[(U)-(E)]^{-1}(W)\right. \\
& \cdot+[(U)-(E)]^{-1}[(U)+(E)] \quad(\mathrm{A} 25) \\
\left(S_{12}^{F}\right)=\left(S_{21}^{F}\right)=- & \left\{(U)-[(U)-(E)]^{-1}(W)\right. \\
& \left.\cdot[(U)-(E)]^{-1}(W)\right\}^{-1} \\
& \cdot\left\{[(U)-(E)]^{-1}(W)[(U)-(E)]^{-1}\right. \\
& \left.\cdot[(U)+(E)]^{-1}+[(U)-(E)]^{-1}(W)\right\}
\end{aligned}
$$

with the matrix coefficients

$$
\begin{aligned}
(E)= & \sum_{n=1}^{3}\left(N_{E}^{(n)}\right) 2\left(R^{(n)}\right) \\
& \cdot\left[\left(R^{(n)}\right)\left(R^{(n)}\right)-(U)\right]^{-1}\left(N_{H}^{(n)}\right)^{-1} \\
(W)= & \sum_{n=1}^{3}\left(N_{E}^{(n)}\right)\left\{2\left(R^{(n)}\right)\left[\left(R^{(n)}\right)\left(R^{(n)}\right)-(U)\right]^{-1}\right. \\
& -(U)\}\left(N_{H}^{(n)}\right)^{-1} \\
\left(N_{E}^{(n)}\right)= & \left(D_{E}^{(n)}\right)^{-1}\left(L_{E}^{(n)}\right) \\
\left(N_{H}^{(n)}\right)= & \left(D_{H}^{(n)}\right)^{-1}\left(L_{H}^{(n)}\right) .
\end{aligned}
$$

\section{REFERENCES}

[1] G. L. Matthaei, L. Young, E. M. T. Jones, Microwave Filters, Impedance-Matching Networks, and Coupling Structures. New York: McGraw-Hill, 1964, ch. 17.

[2] R. F. Fjestad, "Some design considerations and realizations of iris-coupled YIG-tuned filters in the $12-40 \mathrm{GHz}$ region," IEEE Trans. Microwave Theory Tech., vol. MTT-18, pp. 205-212, Apr. 1970.

[3] R. F. Skedd and G. Craven, "Magnetically tunable multisection bandpass filters in ferrite-loaded evanescent waveguide," Electron. Lett.; vol. 3, pp. 62-63, Feb. 1967.

[4] R. V. Snyder, "Stepped-ferrite tunable evanescent filters," IEEE Trans. Microwave Theory Tech., vol. MTT-29, pp. 364-371, Apr. 1981.

[5] J. Meier, "Integrated fin-line millimeter components," IEEE Trans. Microwave Theory Tech., vol. MTT-32, pp. 1209-1216, Dec. 1974

[6] Y. Konishi and K. Uenakada, "The design of a bandpass filter with inductive strip-Planar circuit mounted in waveguide," IEEE Trans. Microwave Theory Tech., vol. MTT-22, pp. 869-873, Oct. 1974.

[7] F. Arndt, J. Bornemann, D. Grauerholz, and R. Vahldieck, "Theory and design of low-insertion loss fin-line filters," IEEE Trans. Microwave Theory Tech., vol. MTT-30, pp. 155-163, Feb. 1982.

[8] Y.-C. Shih and T. Itoh, " $E$-plane filters with finite-thickness septa," IEEE Trans. Microwave Theory Tech., vol. MTT-31, pp. 1009-1012, Dec. 1983.

[9] R. Vahldieck, J. Bornemann, F. Arndt, and D. Grauerholz, "Optimized waveguide $E$-plane metal insert filters for millimeter-wave applications," IEEE Trans. Microwave Theory Tech., vol. MTT-31, pp. $65-69$, Jan. 1983

[10] K. Solbach, "The status of printed millimeter-wave $E$-plane circuits," IEEE Trans. Microwave Theory Tech., vol. MTT-31, pp. 107-121, Feb. 1983.

[11] P. J. Meier, "Integrated finline: The second decade," Microwave J., vol. 28 , no. 11 , pp. $31-54$, Nov. 1985 ; also no. 12 , pp. $30-48$, Dec. 1985.
[12] L. A. Bui, D. Ball, and T. Itoh, "Broad-band millimeter-wave plane bandpass filters," IEEE Trans. Microwave Theory Tech., vol. MTT32 , pp. $1655-1658$, Dec. 1984

[13] Y.MC. Shih, "Design of waveguide $E$-plane filters with all-metal inserts," IEEE Trans. Microwave Theory Tech., vol. MTT-32, pp. 695-704, July 1984.

[14] F. Arndt, J. Bornemann, D. Heckmarı, C. Piontek, H. Semmerow, and $H$. Schueler, "Modal-S-matrix method for the optimum design of inductively direct-coupled cavity filters," Proc. Inst. Elec. Eng., vol. 133, pt. H, pp. 341-350, Oct. 1986

[15] B. K. J. Lax, K. J. Button, and L. M. Roth, "Ferrite phase shifters in rectangular waveguide," J. Appl. Phys., vol. 25, pp. 1413-1421, 1954.

[16] R. E. Collin, Field Theory of Guided Waves. New York: McGraw Hill, ch. 6, pp. 85, 174-179, 198-209, 255.

[17] R. F. Harrington and A. I. Villeneuve, "Reciprocity relationships for gyrotropic media," IRE Trans. Microwave Theory Tech., vol. MTT-6, pp. 308-310, July 1958.

[18] F. E. Gardiol and A. S. Vander Vorst, "Computer analysis of E-plane resonance isolators," IEEE Trans. Microwave Theory Tech., vol. MTT-19, pp. 315-322, Mar. 1974.

[19] F. E. Gardiol, "Anisotropic slabs in rectangular waveguides," IEEE Trans. Microwave Theory Tech., vol. MTT-18, pp. 461-467, Aug. 1970.

[20] A.-M. Khilla and I. Wolff, "Field theory treatment of $H$-plane waveguide junction with triangular ferrite post," IEEE Trans. Microwave Theory Tech., vol. MTT-26, pp. 279-287, 1978.

[21] J. Uher, F. Arndt, and J. Bornemann, "Field theory design of ferrite-loaded waveguide nonreciprocal phase shifters with multisection ferrite or dielectric slab impedance transformers," IEEE Trans. Microwave Theory Tech., vol. MTT-35, pp. 552-560, June 1987.

[22] F. Arndt, J. Bornemann, and R. Vahldieck, "Design of multisection impedance-matched dielectric-slab filled waveguide phase shifter," IEEE Trans. Microwave Theory Tech., vol. MTT-32, pp. 34-38, Jan. 1984.

[23] F. Arndt, A. Frye, M. Wellnitz, and R. Wirsing, "Double dielectric-slab-filled waveguide phase shifter," IEEE Trans. Microwave Theory Tech., vol. MTT-33, pp. 373-381, May 1985.

[24] T. E. Rozzi and W. F. G. Mecklenbräuker, "Wide-band network modeling of interacting inductive irises and steps," IEEE Trans. Microwave Theory Tech., vol. MTT-23, pp. 235-245, Feb. 1975.

[25] M. S. Navarro, T. E. Rozzi, and Y. T. Lo, "Propagation in a rectangular waveguide periodically loaded with resonant irises," IEEE Trans. Microwave Theory Tech., vol. MTT-28, pp. 857-865, Aug. 1980.

[26] R. Mittra and S. Lee, Analytical Techniques in the Theory of Guided Waves. New York: Macmillan, 1971.

[27] H. Schmiedel, "Anwendung der Evolutionsoptimierung bei Mikrowellenschaltungen," Frequenz, vol. 35, pp. 306-310, Nov. 1981.

[28] J. Green, E. Schlömann, F. Sandy, and J. Saunders, "Characterization of the microwave tensor permeability of partially magnetized materials," Semi-annual Rep. RADC-TR-6963, Feb. 1969.

[29] M. Hoffmann, "Die Streumatrix von Rechteckholleitungen mit magnetisierten Ferriteinsätzen endlicher Länge," Arch Elek. Übertragung, vol. 32, pp. 62-68, Feb. 1978.

[30] D. Polder, "On the theory of ferromagnetic resonance," Phil. Mag., vol. 40 , pp. $100-115,1949$.

[31] G. T. Rado, "Theory of the microwave permeability tensor and Faraday effect in nonsaturated ferromagnetic materials," Phys. Rev., vol. 79, p. 529,1953

[32] P. Hlawviczka and A. Mortis, "Gyromagnetic resonance graphical design data," Proc. Inst. Elec. Eng., vol. 11, pp. 665-670, 1963.

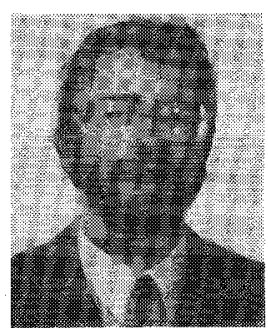

Jaroslaw Uher was born in Klomnice, Poland, on June 2, 1954. He received the M.Sc. degree in electronic engineering from the Technical University of Wroclaw, Poland, in 1978 and the Dr.-Ing. degree in microwave engineering from the University of Bremen, Bremen, West Germany, in 1987.

From 1978 to 1982 his work dealt with planar ferrite engineering at the Institute of Electronic Technology, Technical University of Wroclaw. Since 1983, he has been with the Microwave 
Department of the University of Bremen, where his present research activities involve the solving electromagnetic field problems of ferrimagnetic slab discontinuities in waveguide structures and the design of ferrite control components and filters.

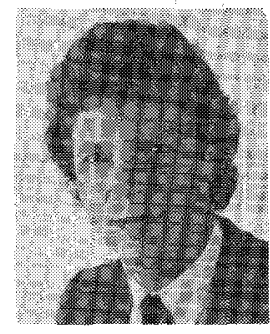

Jens Bornemann was born in Hamburg, West Germany, on May 26, 1952. He received the Dipl-Ing. and Dr.-Ing. degrees, both in electrical engineering, from the University of Bremen, Bremen, West Germany, in 1980 and 1984, respectively.

From 1980 to 1983 , he was a Research Assistant in the Microwave Department of the University of Bremen, where he worked on quasi-planar waveguide configurations and computer-aided $E$-plane filter design. After a twoyear period as a consulting engineer, he joined the University of Bremen again, in 1985, where he was employed at the level of Assistant Professor. In April 1988 he became an Associate Professor at the University of Victoria, B.C., Canada. His current research activities include microwave and millimeter-wave integrated circuit components and systems, problems of electromagnetic field theory, and numerical methods.

Dr. Bornemann was one of the recipients of the A. F. Bulgin Premium of the Institution of Electronic and Radio Engineers (1983).

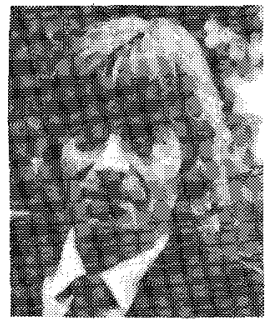

Fritz Arndt (SM'83) received the Dipl.-Ing., Dr.Ing., and Habilitation degrees from the Technical University of Darmstadt, West Germany, in 1963,1968 , and 1972, respectively.

From 1963 to 1972 he worked on directional couplers and microstrip techniques at the Technical University of Darmstadt. Since 1972, he has been a Professor and Head of the Microwave Department at the University of Bremen, West Germany. His research activities involve field problems of waveguide, finline, and optical waveguide structures, antenna design, and scattering structures.

Dr. Arndt is member of the VDE and VTG (Germany). He received the NTG award in 1970, the A. F. Bulgin Award (together with three coauthors) from the Institution of Radio and Electronic Engineers in 1983, and the best paper award of the antenna conference JINA 1986 (France). 